\title{
Cord Blood-derived Expanded Natural Killer Cells PNK-007
}

National Cancer Institute

\section{Source}

National Cancer Institute. Cord Blood-derived Expanded Natural Killer Cells PNK-007. NCI

Thesaurus. Code C128560.

A population of allogeneic lytic natural killer (NK) cells derived from human umbilical cord blood (UCB), with potential cytotoxic activity. Hematopoietic stem cells (HSC) are isolated from human UCB; this is followed by ex vivo differentiation into mature, highly lytic, NK cells, and expansion. Upon administration, the CB-derived expanded NK cells PNK-007 may lyse cancer cells. 